\title{
Carcinoma Cell Line Discrimination in Microscopic Images Using Unbalanced Wavelets
}

\author{
Furkan Keskin, Alexander Suhre, Tülin Erşahin, Rengül Çetin-Atalay and A. Enis Çetin, Fellow, IEEE
}

\begin{abstract}
Cancer cell lines are widely used for research purposes in laboratories all over the world. In this paper, we present a novel method for cancer cell line image classification, which is very costly by conventional methods. The aim is to automatically classify 14 different classes of cell lines including 7 classes of breast and 7 classes of liver cancer cells. Microscopic images containing irregular carcinoma cell patterns are represented by randomly selected subwindows which possibly correspond to foreground pixels. For each subwindow, a correlation descriptor utilizing the fractional unbalanced wavelet transform coefficients and several morphological attributes as pixel features is computed. Directionally selective textural features are preferred primarily because of their ability to characterize singularities at multiple orientations, which often arise in carcinoma cell lines. A Support Vector Machine (SVM) classifier with Radial Basis Function (RBF) kernel is employed for final classification. Over a dataset of 280 images, we achieved an accuracy of $88.2 \%$, which outperforms the classical correlation based methods.
\end{abstract}

Index Terms-Carcinoma cell line, microscopic image processing, correlation descriptor, unbalanced wavelet.

\section{INTRODUCTION}

A UTOMATIC classification of biomedical images is an emerging field, despite the fact that there is a long history of image recognition techniques [1]. Automated classification of carcinoma cells through morphological analysis will greatly improve and speed up cancer research conducted using established cancer cell lines as in vitro models. Distinct morphologies of different types and even sub-types of cancer cells reflect, at least in part, the underlying biochemical differences, i.e., gene expression profiles. Moreover, the morphology of cancer cells can infer invasivenes of tumor cell and hence the metastatic capability. In addition, an automated morphological classification of cancer cells will enable the correct detection and labelling of different cell lines. Predicting labels of cancer cell lines in a fast and accurate manner via a pattern classification approach will greatly enhance biologists' ability to identify different types of cell lines without the need to scrutinize each and every microscopic image one by one. Although cell lines are being used widely as in vitro models in cancer research and drug development, mislabeling cell lines or failure to recognize any contamination may lead to misleading results. Short tandem repeat (STR) analysis is being used as a standard for the authentication of human cell lines. However, this process takes a long time and has to be

F. Keskin, A. Suhre and A. E. Cetin are with the Department of Electrical and Electronics Engineering, Bilkent University, Bilkent, Ankara, 06800, Turkey. e-mail: keskin@ee.bilkent.edu.tr

T. Ersahin and R. Çetin-Atalay are with the Department of Molecular Biology and Genetics, Bilkent University, Bilkent, Ankara, 06800, Turkey. carried out by an expert. Automated analysis, on the other hand, will provide the scientists a fast and easy-to-use tool that they can use in their own laboratories to verify their cell lines.

In this study, discrimination of 14 classes of biomedical images is achieved, which are all images of cancer cell lines. The dataset in hand consists of two types of cancer cell lines, namely breast cancer and liver cancer (hepatocellular carcinoma). The breast cancer cell lines used in our study are BT-20, Cama-1, MDA-MB-157, MDA-MB-361, MDA-MB-453, $M D A-M B-468$ and $T 47 D$, while the liver cancer cell lines are Focus, Hep40, HepG2, Huh7, mv, PLC and SkHepl. Sample images from breast cancer and liver cancer cell lines are shown in Figures 1-8.

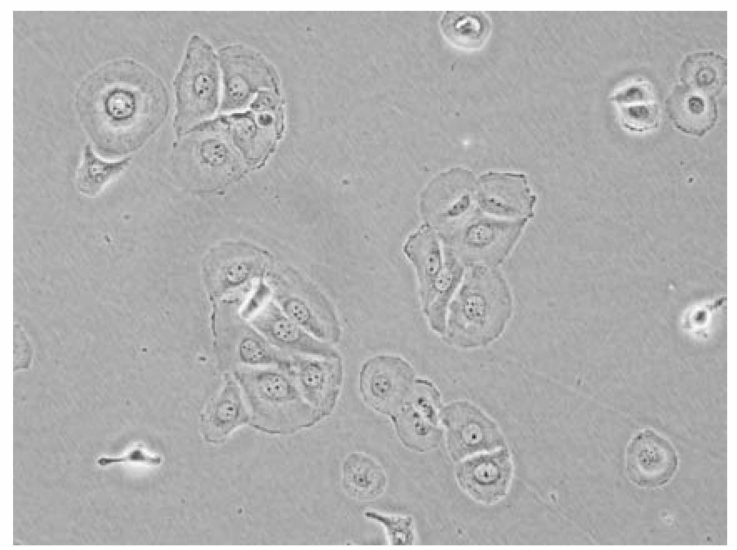

Fig. 1: Sample image from BT-20 class

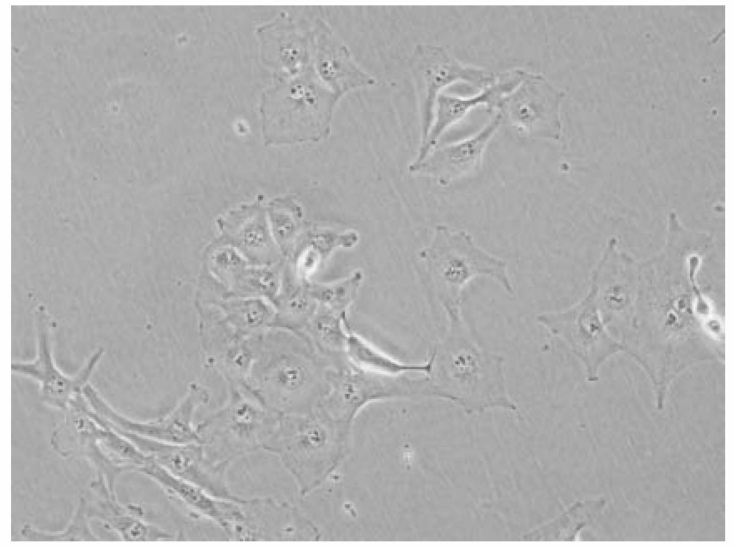

Fig. 2: Sample image from Focus class

We adopt a correlation based approach by exploiting pixellevel attributes to construct local region descriptors encoding 


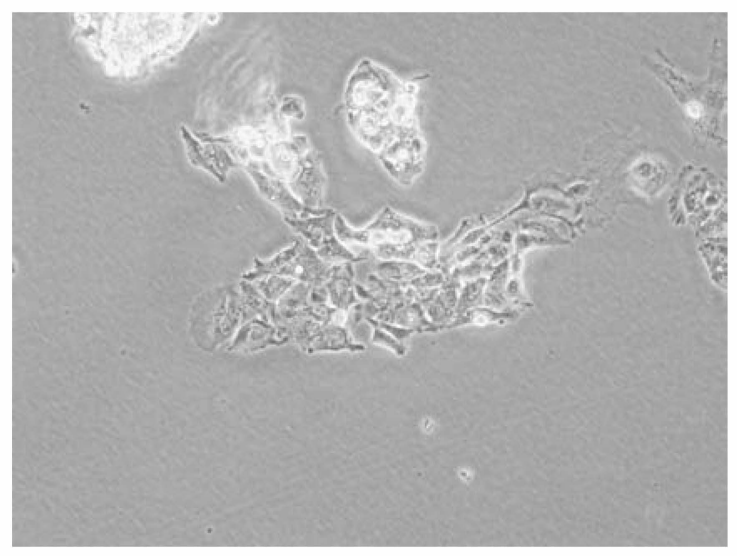

Fig. 3: Sample image from $H e p G 2$ class

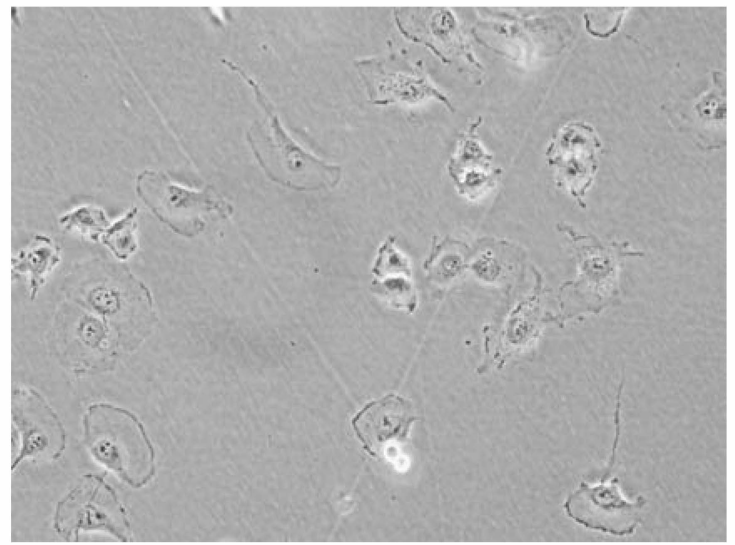

Fig. 4: Sample image from $M D A-M B-157$ class

correlations of several attributes inside a region of interest. Pixel attributes are extracted using directional difference scores and directionally selective fractional wavelet transform. Since background regions occur frequently in a cancer cell line image, we randomly sample subwindows from the foreground image regions a ter foreground-background segmentation and each microscopic image is represented by correlation matrices of certain number of subwindows sampled randomly from the whole image. Finally, an SVM classifier with RBF kernel is trained to learn the class boundaries.

This paper is organized as follows. In Section 2, feature extraction steps are described comprising image decomposition method by directionally selective fractional wavelet transform, directional difference score computation and correlation matrix construction. In Section 3, SVM based correlation matrix classification algorithm is explained along with the foregroundbackground segmentation by EM algorithm and random subwindow selection. Section 4 presents the experimental results and Section 5 concludes the paper.

\section{FEATURE EXTRACTION}

\section{A. Directionally Selective Fractional Wavelet Transform}

Microscopic cancer cell line images contain significant amount of oriented singularities. To characterize the singularities at multiple orientations, we propose the directionally selective fractional wavelet transform (DS-FWT) for analysis

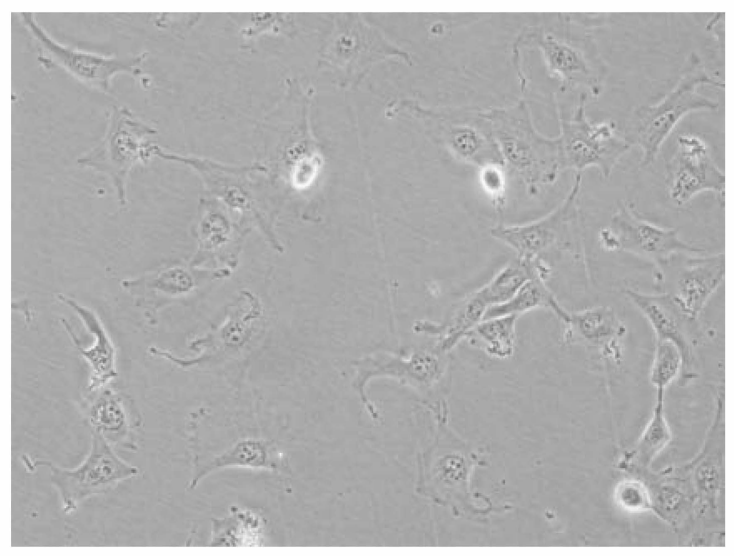

Fig. 5: Sample image from $M V$ class

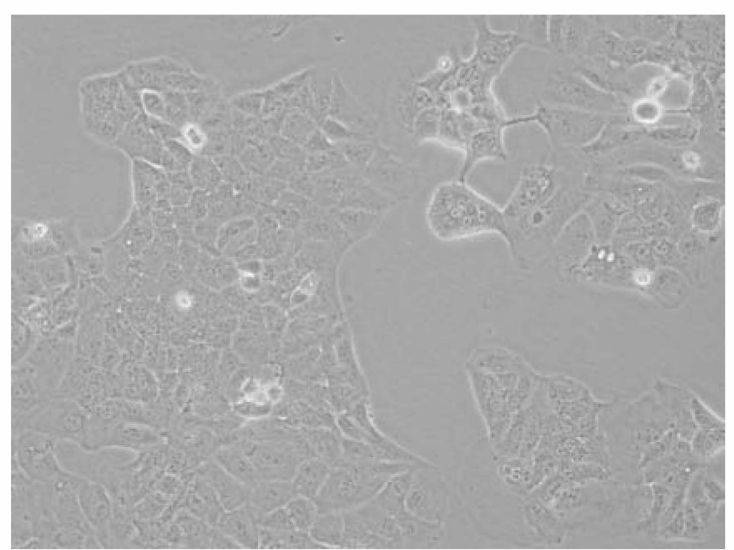

Fig. 6: Sample image from $P L C$ class

of microscopic images [2]. Fractional wavelet decomposition [3] is based on two-channel unbalanced lifting structures whereby it is possible to decompose a given discrete-time signal $x[n]$ sampled with period $T$ into two sub-signals $x_{1}[n]$ and $x_{2}[n]$ whose average sampling periods are $p T$ and $q T$, respectively. Fractions $p$ and $q$ are rational numbers satisfying the condition: $1 / p+1 / q=1$. In FWT, a structure similar to the lazy filterbank forwards every $p^{\text {th }}$ sample of the original signal to the upper-branch and remaining $p-1$ samples out of $p$ samples go to the lower branch. An example of an unbalanced li ting structure with $p=3: 1$ and $q=3: 2$ is shown in Figure 9.

In Figure 9, upper branch sub-signal samples $x_{L}[n]$ are updated using the neighboring lower-branch samples as

$x_{L}[n]=\frac{1}{9} x[3 n-2]+\frac{2}{9} x[3 n-1]+\frac{3}{9} x[3 n]+\frac{2}{9} x[3 n+1]+\frac{1}{9} x[3 n+2]$

where $x[n]$ is the original input signal to the filterbank. Lower branch detail signal samples are predicted from the upper branch and the difference is transmitted to the output lower branch:

$x_{H}[n]= \begin{cases}x\left[\frac{3 n+2}{2}\right]-\frac{2 x_{L}\left[\frac{n}{2}\right]+x_{L}\left[\frac{n}{2}+1\right]}{3}, & \mathrm{n} \text { is even } \\ x\left[\frac{3 n+1}{2}\right]-\frac{2 x_{L}\left[\frac{n+1}{2}\right]+x_{L}\left[\frac{n-1}{2}\right]}{3}, & \mathrm{n} \text { is odd }\end{cases}$ 


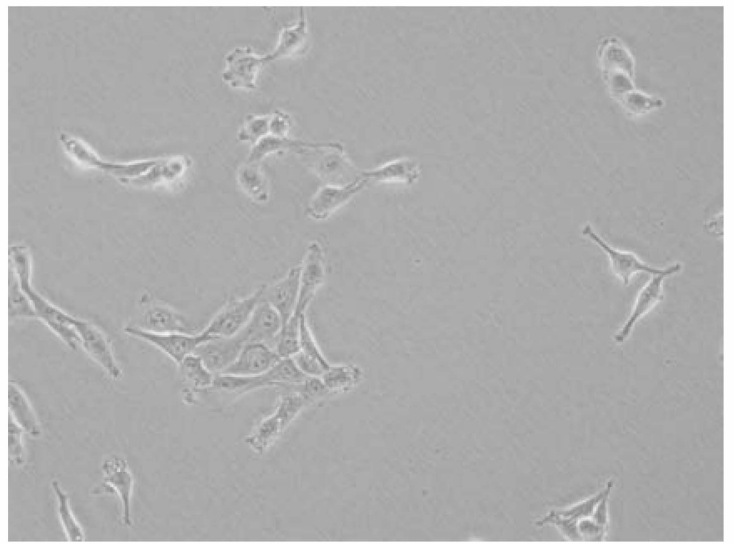

Fig. 7: Sample image from SkHepl class

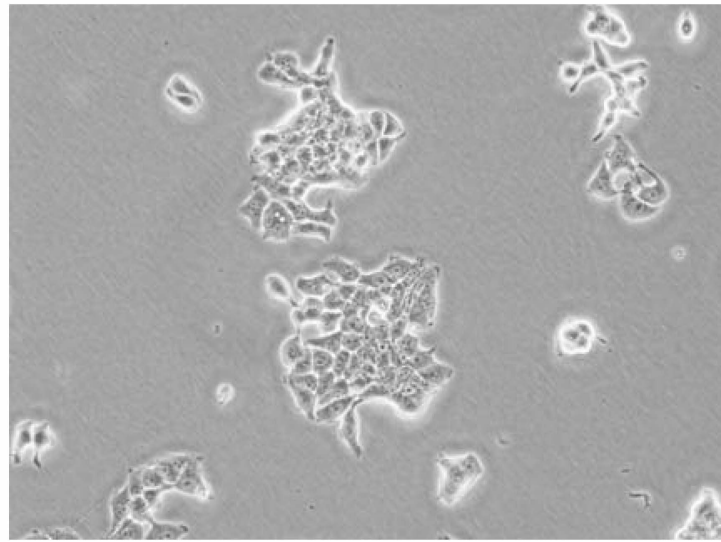

Fig. 8: Sample image from $T 47 D$ class

We extended the fractional wavelet transform to twodimensional signals in a non-separable manner and use it to extract directionally selective textural features from cancer cell line images. 2-D DS-FWT unbalanced lifting structure with $p=9: 1$ and $q=9: 8$, which produces five directional subbands, is shown in Figure 10.

In this study, DS-FWT is used with $p=25: 1$ and $q=$ $25: 24$. A 2-D image is divided into $5 \times 5$ nonoverlapping cells and eight values corresponding to eight orientations between 0 and $\pi$ are computed inside each cell. With $I(x, y)$ denoting the center pixel of the cell, fractional wavelet features can be computed by taking differences between the center pixel and the weighted region sums as follows:

$$
\begin{aligned}
& D_{1}(x, y)=\left|I(x, y)-\sum_{\substack{1 \geq i \geq-1 \\
2 \geq j \geq-2}} w_{i j} I(x+i, y+j)\right| \\
& D_{2}(x, y)=\left|I(x, y)-\sum_{\substack{1 \geq i \geq-1 \\
2 \geq j>-2 \\
0 \geq i j}} w_{i j} I(x+i, y+j)\right|
\end{aligned}
$$

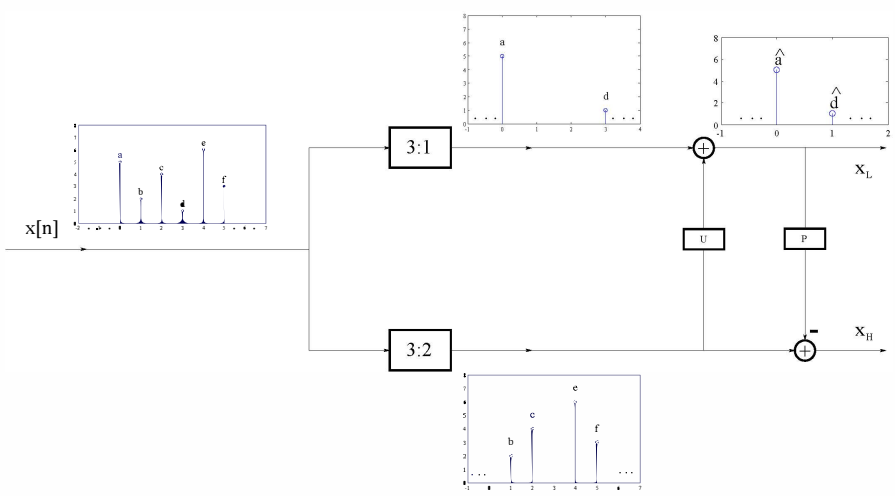

Fig. 9: Unbalanced lifting scheme with $p=3: 1$ and $q=3: 2$ downsampling ratios.

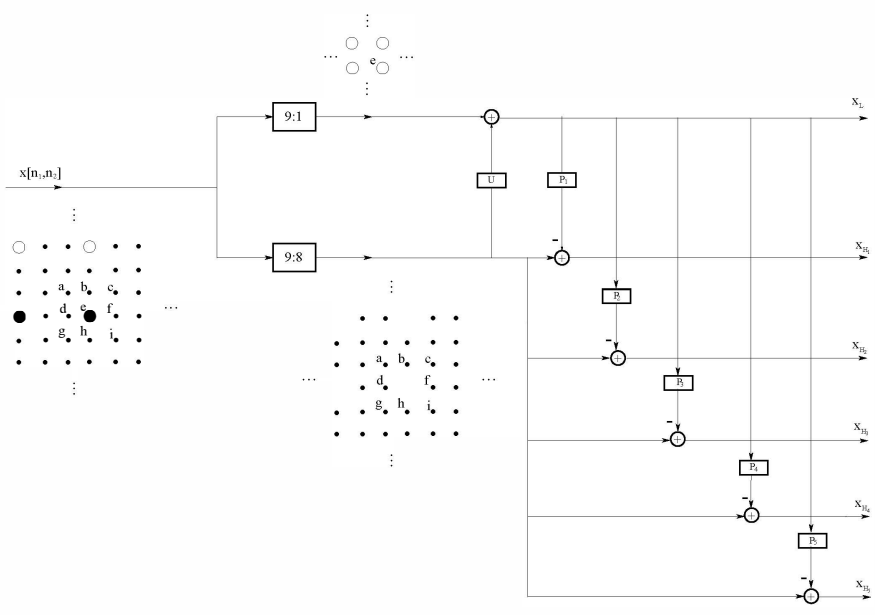

Fig. 10: 2-D non-separable extension of the unbalanced lifting structure with $p=9: 1$ and $q=9: 8$.

$$
\begin{aligned}
& D_{3}(x, y)=\left|I(x, y)-\sum_{\substack{2 \geq i \geq-2 \\
2 \geq j \geq-2 \\
0>i j}} w_{i j} I(x+i, y+j)\right| \\
& D_{4}(x, y)=\left|I(x, y)-\sum_{\substack{2 \geq i \geq-2 \\
1 \geq j \geq-1 \\
0>i j}} w_{i j} I(x+i, y+j)\right|
\end{aligned}
$$

where $D_{i}(x, y), i=1 \ldots 8$, represents the directional feature for the $i^{t h}$ direction, $w_{i j}$ denotes the weight associated with the corresponding pixel in the neighborhood of the center pixel and $\sum_{i, j} w_{i j}=1$. The subband images $D_{i}(x, y)$ for $i=5 \ldots 8$ are computed in a similar manner. They represent $\pi / 4$ counterclockwise rotated versions of the differences $D_{i}(x, y), i=1 \ldots 4$, respectively. Each $D_{i}$ can be regarded as a directional detail subband whose size is five times smaller than that of the original size. To obtain an approximation subband the same size as the detail subbands, we decimate the image by a factor of five using anti-aliasing filters and 
obtain an approximation image $I_{a}(x, y)$. Thus, we obtain 9 attributes for each pixel $(\mathrm{x}, \mathrm{y})$ of the image $I_{a}$, which are the intensity $I_{a}(x, y)$ and 8 values $D_{i}(x, y)$ obtained from fractional wavelet transformation. These values will be used as features in correlation matrix computation.

\section{B. Directional Differences}

In order to account for the large morphological variation of the images in our dataset, we evaluated differences between pixels in various directions. Consider a point $p_{1}$ on a twodimensional function $I(x, y)$. Now consider a second point $p_{2}$. The Euclidean distance between $p_{1}$ and $p_{2}$ is $d$ and $p_{2}$ lies on line that has an orientation of angle $\alpha$ with respect to the $x$-coordinate, i.e., $p_{2}$ lies on a circle, which's center point is $p_{1}$ and has a radius $d$. The difference between $p_{1}$ and $p_{2}$ can be written as

$$
T(d, \alpha)=|I(x, y)-I(x+d \cdot \cos \alpha, y+d \cdot \sin \alpha)| .
$$

Now consider we want to compute a couple of difference values for equidistant concentric circles where the largest circle has radius $R$ and the smallest has radius $R / A$, where $A$ is an integer with values ranging from $[1, R]$. When the parameters $R$ and $A$ are fixed, we can rewrite the above equation as

$$
T(i, \alpha)=\left|I(x, y)-I\left(x+i \frac{R}{A} \cdot \cos \alpha, y+i \frac{R}{A} \cdot \sin \alpha\right)\right|,
$$

where $i \in 1,2, \ldots, A$. We can compute a score for each $\alpha$ value by computing a function with respect to $i$, as

$$
s_{\alpha}=\mathcal{F}_{\rangle}(T(i, \alpha)) \text {. }
$$

For example, $\mathcal{F}_{\rangle}$can be the median function. In that case $s_{\alpha}$ is simply the median of all the differences between the center pixel and the points at distances $i \frac{R}{A}$ at the fixed orientation $\alpha$. We use these scores as features in correlation matrix computation. Three different functions, namely median, max and mean functions, are employed for $\mathcal{F}\rangle$ in this study. For each image $I_{a}(x, y)$ obtained in Section II-A, 8 output images of the same size are generated as the result of the function $\mathcal{F}$, corresponding to 8 different orientations when the radius $d$ is chosen as 5 in the experiments. Hence, in addition to DSFWT features, each pixel $(\mathrm{x}, \mathrm{y})$ of the image $I_{a}$ has 8 attributes, which denote the scores $s_{\alpha}$ for 8 different $\alpha$ values.

\section{Correlation Matrices for Cell Line Description}

Successfully employed in texture classification [4], pedestrian detection [5] and flame detection [6], covariance descriptors enable the combination of different features over an image region of interest. Given an intensity image I of size $m \times n$, we define a mapping $\phi$ from image domain to feature domain as

$$
F(x, y)=\phi(I, x, y)
$$

where each pixel $(\mathrm{x}, \mathrm{y})$ is mapped to a set of features and $\mathrm{F}$ is the $m \mathrm{x} n \mathrm{x} d$ dimensional feature function. For a given subwindow $\mathrm{R}$ consisting of $\mathrm{n}$ pixels, let $\left(\mathbf{f}_{\mathbf{k}}\right)_{k=1 \ldots n}$ be the $d$-dimensional feature vectors extracted from $\mathrm{R}$. Then, the covariance matrix of region $\mathrm{R}$ can be computed as

$$
\mathbf{C}=\frac{\mathbf{1}}{\mathbf{n}-\mathbf{1}} \sum_{\mathbf{k}=\mathbf{1}}^{\mathbf{n}}\left(\mathbf{f}_{\mathbf{k}}-\mu\right)\left(\mathbf{f}_{\mathbf{k}}-\mu\right)^{\mathbf{T}}
$$

where $\mu$ is the mean of the feature vectors inside the region $\mathrm{R}$. The covariance matrix is symmetric positive-definite and of size $d x d$. There exists a very efficient multiplier-less implementation of covariance descriptors, called co-difference matrices, which have been shown to yield comparable performances to the original ones [7].

Correlation descriptors are obtained by normalizing covariance matrices. Correlation matrix estimation is performed as [8]

$$
\hat{\mathbf{C}}(\mathbf{i}, \mathbf{j})= \begin{cases}\sqrt{\mathbf{C}(\mathbf{i}, \mathbf{j})}, & \text { if } i=j \\ \frac{\mathbf{C}(\mathbf{i}, \mathbf{j})}{\sqrt{\mathbf{C}(\mathbf{i}, \mathbf{i}) \mathbf{C}(\mathbf{j}, \mathbf{j})},} & \text { otherwise }\end{cases}
$$

With

$$
\mathbf{D}(\mathbf{x}, \mathbf{y})=\left[\begin{array}{llll}
D_{1}(x, y) & \ldots & D_{8}(x, y)
\end{array}\right]
$$

and

$$
\mathbf{s}_{\alpha}^{\mathbf{k}}(\mathbf{x}, \mathbf{y})=\left[s_{\alpha_{1}}^{k}(x, y) \ldots s_{\alpha_{\mathbf{g}}}^{k}(x, y)\right]
$$

where $\alpha_{1} \ldots \alpha_{8}$ correspond to the eight orientations of directional difference score estimation and $k=1,2,3$ denote, respectively, the median, $\max$ and mean functions $\mathcal{F}_{\rangle}$in Section II-B, feature mapping functions employed in this study are

$$
\begin{aligned}
& \phi_{1}(I, x, y)=\left[\begin{array}{lll}
I_{a}(x, y) & \mathbf{D}(\mathbf{x}, \mathbf{y}) & \mathbf{s}_{\alpha}^{\mathbf{1}}(\mathbf{x}, \mathbf{y})
\end{array}\right]^{T}, \\
& \phi_{2}(I, x, y)=\left[\begin{array}{lll}
I_{a}(x, y) & \mathbf{D}(\mathbf{x}, \mathbf{y}) & \mathbf{s}_{\alpha}^{\mathbf{2}}(\mathbf{x}, \mathbf{y})
\end{array}\right]^{T}, \\
& \phi_{3}(I, x, y)=\left[\begin{array}{lll}
I_{a}(x, y) & \mathbf{D}(\mathbf{x}, \mathbf{y}) & \mathbf{s}_{\alpha}^{\mathbf{3}}(\mathbf{x}, \mathbf{y})
\end{array}\right]^{T}, \\
& \phi_{4}(I, x, y)=\left[I_{a}(x, y) \quad\left|I_{x}\right| \quad\left|I_{y}\right| \quad\left|I_{x x}\right| \quad\left|I_{y y}\right|\right]^{T}
\end{aligned}
$$

where $\left|I_{x}\right|$ and $\left|I_{x x}\right|$ denote the first- and second-order derivatives at $(x, y)$ of the image $I_{a}$.

\section{Classification Using a Multiclass SVM}

The images in our dataset show a large amount of background pixels. Clearly, the background is not discriminative. Therefore, we address the issue of segmenting the images into foreground and background before classification. For our dataset a simple thresholding scheme is not sufficient for segmentation, since foreground pixels have a large variance and may therefore have values higher and lower than the background pixels. We modeled the image as a mixture of two Gaussians, representing the foreground and background pixels, respectively. Using this model, an Expectation-Maximization (EM) algorithm was applied for segmentation. 
Since it is necessary to focus on foreground-like regions in carcinoma cell line images, $s$ analysis square windows are randomly selected, as in [9], from each image with the two constraints: the percentage of the foreground pixels in the selected region of an image must be above 50 and the variance of the selected region must exceed an imagedependent threshold, which is the variance of the whole image.

For each subwindow, a covariance matrix is computed using Equation (11) for each of the feature mapping functions in (15)-(18). The image signature is composed of $s$ covariance matrices of the same size. Each class is represented by $s \mathrm{x} \#$ (images in each class) covariance matrices. Covariance matrices are symmetric positive-definite and do not lie in the Euclidean space; so, they are vectorized resulting in $d(d+1) / 2$-dimensional vectors for $d x d$ matrices. A multiclass SVM classifier is trained with RBF kernel in the $d(d+1) / 2$ dimensional vector space using the training points. SVM algorithm is implemented using LIBSVM library [10]. For each test subwindow, the corresonding covariance descriptor is vectorized and fed into the trained SVM model for prediction. Therefore, there exist $s$ labels for each microscopic image corresponding to $s$ subwindows, and the image in question is assigned the label that gets the majority of votes among $s$ labels. The above process is re-executed using correlation matrices instead of covariance matrices.

\section{EXPERIMENTS}

The dataset used in this study consists of 280 microscopic human carcinoma cell line images with each of the 14 classes having 20 images. All the images in the dataset were acquired at 20x magnification. The size of each image is $3096 \times 4140$ pixels. 7 classes belong to breast cancer cell lines and the other classes belong to liver cancer. Breast cancer cells tend to be smaller and round whereas liver cancer cells tend to be more spread out and have pods. Moreover, breast cells generally do not contain dots within the cytoplasm.

We adopt a 20-fold cross-validation strategy for the experiments. The dataset is divided into 20 disjoint subsets and each subset consisting of 14 images is used exactly once as the test set. For $k=1 \ldots 20, k^{t h}$ subset is formed by taking the $k^{\text {th }}$ indexed image of each class. We run 20 experiments, choosing each image as the test image only once for each class, and obtain the average image classification accuracy over 20 runs. The number of selected random subwindows is taken to be $s=100$. We perform the above experiment for both covariance and correlation matrices, and for four different mapping functions in (15)-(18). SVM RBF kernel parameters are chosen as $\gamma=0.5$ and $C=1000$. Experimental results are shown in Table I.

It can be seen from Table I that correlation matrix-based method outperforms the covariance method for all mapping functions. This can be attributed to the normalization performed in correlation computation. Unbalanced wavelet features based classification methods (15)-(17) have higher accuracies than the classical covariance/correlation method in (18). It is clearly demonstrated via experiments that image classification accuracy can be enhanced by exploiting the directional information through the use of DS-FWT features and directional scores obtained by median, max and mean functions.

\begin{tabular}{|c|c|c|}
\hline $\begin{array}{c}\text { Feature } \\
\text { mapping } \\
\text { function }\end{array}$ & $\begin{array}{c}\text { Covariance } \\
\text {-based } \\
\text { classification }\end{array}$ & $\begin{array}{c}\text { Correlation } \\
\text {-based } \\
\text { classification }\end{array}$ \\
\hline$\phi_{1}(I, x, y)$ & 82.5 & 85 \\
\hline$\phi_{2}(I, x, y)$ & 84.3 & 87.1 \\
\hline$\phi_{3}(I, x, y)$ & 87.1 & 88.2 \\
\hline$\phi_{4}(I, x, y)$ & 73.2 & 80 \\
\hline
\end{tabular}

TABLE I: Average classification accuracies (in \%) of carcinoma cell line images over 20 runs using SVM with RBF kernel

\section{CONClusion}

In this paper, fractional wavelet transform and region covariance framework are successfully applied to classify the cancer cell line images. We demonstrate that automatic classification of microscopic carcinoma cell line images can be reliably performed using directionally selective fractional wavelet transform (DS-FWT) and correlation descriptors. Fractional wavelet transformation is achieved by using unbalanced lifting structures where sampling intervals of upper and lower branches are different. Covariance and correlation descriptors are computed for features extracted from DS-FWT subbands and directional difference scores. Promising classification results are obtained with the experiments, which reveal the ability of the proposed features to characterize breast and liver carcinoma cell line textures.

\section{REFERENCES}

[1] M. Dundar, S. Badve, V. Raykar, R. Jain, O. Sertel, and M. Gurcan, "A multiple instance learning approach toward optimal classification of pathology slides," in Pattern Recognition (ICPR), 2010 20th International Conference on, August 2010, pp. 2732 -2735.

[2] F. Keskin and A. E. Cetin, "Directionally selective fractional wavelet transform using a 2-d nonseparable unbalanced lifting structure," in MUSCLE International Workshop on Computational Intelligence for Multimedia Understanding, December 13-15 2011.

[3] K. Habiboglu, Y.H.; Kose and A. E. Cetin, "Fractional wavelet transform using an unbalanced lifting structure," in Independent Component Analyses, Wavelets, Neural Networks, Biosystems, and Nanoengineering IX, Proc. SPIE 8058, 805805, 2011.

[4] O. Tuzel, F. Porikli, and P. Meer, "Region covariance: A fast descriptor for detection and classification," in In Proc. 9th European Conf. on Computer Vision, 2006, pp. 589-600.

[5] — , "Pedestrian detection via classification on riemannian manifolds," Pattern Analysis and Machine Intelligence, IEEE Transactions on, vol. 30, pp. 1713-1727, 2008.

[6] Y. Habiboglu, O. Gunay, and A. Cetin, "Flame detection method in video using covariance descriptors," in Acoustics, Speech and Signal Processing (ICASSP), 2011 IEEE International Conference on, May 2011, pp. $1817-1820$.

[7] H. Tuna, I. Onaran, and A. Cetin, "Image description using a multiplierless operator," Signal Processing Letters, IEEE, vol. 16, no. 9, pp. 751 -753 , sept. 2009.

[8] Y. H. Habiboglu, O. Gunay, and A. E. Cetin, "Real-time wildfire detection using correlation descriptors," in 19th European Signal Processing Conference (EUSIPCO 2011), Special Session on Signal Processing for Disaster Management and Prevention, 2011.

[9] R. Maree, P. Geurts, J. Piater, and L. Wehenkel, "Random subwindows for robust image classification," in Computer Vision and Pattern Recognition, 2005. CVPR 2005. IEEE Computer Society Conference on, vol. 1, June 2005 , pp. $34-40$ vol. 1 . 
[10] C.-C. Chang and C.-J. Lin, "LIBSVM: A library for support vector machines," ACM Transactions on Intelligent Systems and Technology, vol. 2, pp. 27:1-27:27, 2011, software available at http://www.csie.ntu.edu.tw/ cjlin/libsvm. 